\title{
Communication
}

\section{The Dynamics of Domain Wall Motion in Spintronics}

\author{
Diego Bisero $\mathbb{D}$ \\ Dipartimento di Fisica e Scienze della Terra, Università degli studi di Ferrara, Via Saragat 1, \\ I-44122 Ferrara, Italy; bisero@fe.infn.it
}

Received: 26 May 2020; Accepted: 24 June 2020; Published: 2 September 2020

check for updates

\begin{abstract}
A general equation describing the motion of domain walls in a magnetic thin film in the presence of an external magnetic field has been reported in this paper. The equation includes all the contributions from the effects of domain wall inertia, damping and stiffness. The effective mass of the domain wall, the effects of both the interaction of the DW with the imperfections in the material and damping have been calculated.
\end{abstract}

Keywords: magnetic thin films; domain walls; magnetic nanostructures; theoretical models and calculations

\section{Introduction}

Domain wall (DW) motion can be induced by external magnetic fields or by spin polarized currents. Domain wall (DW) propagation [1-4] in laterally patterned magnetic thin films [5-8] holds promise for both fundamental research and technological applications and has attracted much attention because of its potential in data storage technology and logic gates, where data can be encoded nonvolatilely as a pattern of magnetic DWs traveling along magnetic wires [9]. Up to now, a remarkable part of measurements with injected spin polarized currents have been performed on systems with the magnetization direction in-plane. Nanosized wires containing DWs driven by spin-transfer torque are the basis, at the moment, of the racetrack memory concept development. However, the induction of DW displacement requires high spin-current densities, unsuitable to realize low power devices. Up to now, materials with low damping and magnetocrystalline anisotropy [10-12] have been the privileged choice of the research community to study DW propagation. Materials with low damping are more susceptible to changes of the DW spin structure, yielding significant effects on the DW dynamics. As a matter of fact, both DW velocity and DW pinning are affected by the microscopic DW spin structure, which in turn depends crucially on the physical and chemical properties of the specific material used. Moreover, a DW driven by an electrical current moves mainly through the angular momentum transfer; therefore multiple DWs are pushed in the same direction.

The magnetic-field-driven DW motion presents some drawback too; the main cause of DW propagation is energy dissipation and the relative speed is proportional to the energy dissipation rate. The magnetic field could eliminate unfavorable domains, instead of moving different DWs synchronously.

An alternative approach could be the use of a spinwave spin current, exploiting thermal gradients, to control DW motion. From a microscopic point of view, magnons occupying hotter regions diffuse to the colder ones forming a magnon spin current, which passes through a DW and exerts a torque on it. However, this is a relatively new and still not fully explored area.

In view of complex and multi-purpose spintronic devices, the three main driving mechanisms for DW motion (magnetic field, electric currents and spin wave currents) could be integrated on the same chip, preferring one mechanism over another depending on the specific properties of the DW that one needs to preserve or enhance. A fourth alternative approach for DW motion is proposed: 
the combination of laser pulses with current pulses. This would provide a new degree of freedom, represented by laser polarization, and could originate helicity-dependent DW motion, allowing for a drastic reduction in the threshold current necessary to trigger the spin torque effect. Moreover, the use of laser pulses would also introduce the possibility of generating local thermal gradients, with a relative injection of spin wave currents, enabling an alternative class of low-power photonic/spintronic devices beyond the conventional approach. This would sketch an energy-efficient strategy, highlighting the development of new classes of low-power optoelectronic memory and logic devices, integrating photonics and electronics.

In this complex context, any theoretical knowledge that allows the description of the dynamics of the motion of the domain walls in magnetic nanomaterials becomes of paramount importance. Faced with an enormous amount of data and several successful theoretical approaches to specific problems concerning laterally confined materials, a general equation describing the dynamics of the motion of a Bloch DW is still missing. This article proposes an equation that discusses the motion of DWs in the presence of an external magnetic field, calculated in c.g.s. units, providing a powerful tool for chemists and physicists who want to optimize the design of new generation materials and devices. The external magnetic field could be easily replaced by an effective field in the equation, simulating for example, the case of injected currents and spin waves.

\section{The Theoretical Model and the Equation of Motion}

DW motion is supposed to be a process slower than a coherent magnetization reversal because it concerns a sequential, rather than a simultaneous, modification of the orientation of the spin. Therefore, in order to obtain an equation of motion for a DW, one should include the effects of DW inertia, damping and stiffness.

DW inertia: consider an infinite Bloch wall in the $x y$ plane and a field $H$ parallel to the magnetization $M$ on one side of the wall. Let's call $\alpha$ the angle between the direction of $M$ at the zero field and the direction of $M$ in the applied field, $\psi$ the angle between the direction of $M$ at the zero field and the direction of $H$ (see Figure 1). The effect of $H$ is to generate a torque on the magnetization in the wall, causing a precession in the field with angular velocity described by

$$
\dot{\alpha}=-\gamma H \sin \psi .
$$

The parameter $\gamma$ in Equation (1) describes the rate of precession around $H$, as included in the well-known Landau-Lifshitz equation of motion [13,14]. The magnetization is forced to turn in the plane of the wall by this movement, due to the creation of a demagnetizing field within the wall. Taking small values of $\alpha$ one obtains the angular velocity:

$$
\dot{\psi}=M C \alpha
$$

where $C$ is a constant determined by the anisotropy, the exchange interaction and the demagnetizing field. Equation (2) can be expressed as a function of the wall velocity $v=\mathrm{d} \xi / \mathrm{d} t$ (with $\xi$ perpendicular to the $x y$ plane) as the DW preserves its structure during displacement:

$$
\frac{\partial \psi}{\partial \xi} v=M C \alpha
$$

Differentiating (3) with respect to time and using (1) one gets

$$
\frac{\partial \psi}{\partial \xi} \frac{v}{\gamma}=-M C H \sin \psi
$$


The wall must possess an effective mass, as demonstrated by Equation (4), showing that the acceleration of the wall is proportional to the applied pressure $M H$. The effective mass $m_{w}$ for square centimeter is

$$
m_{w}=\frac{1}{2 \pi E_{w}^{2}} \sqrt{\frac{K}{A}}
$$

where $E_{w}, K$ and $A$ are the wall energy density, the anisotropy constant and the exchange constant, respectively. The effective mass turns out to be essentially proportional to its thickness.

DW damping: it can be deduced by the L-L equation [13]. The damping constant cannot be directly equal to $\lambda$ appearing in the L-L equation due to the finite size of the DW. The damping constant for a Bloch wall then becomes [15]:

$$
\delta=\frac{4 \lambda}{\gamma^{2}} \sqrt{\frac{K}{A}} .
$$

In this case, the damping $\delta$ turns out to be inversely proportional to the wall thickness. This situation is different from that which would occur in a bulk material, where the eddy currents due to variations in magnetization give a proportional contribution to the thickness, becoming negligible in the case of thin films.

DW stiffness: this contribution emerges from the interaction of the DW with the imperfections in the material and must be inserted into the equation of motion for a DW. The stiffness $\eta$ is a parameter depending on the local structural and chemical properties of the material and cannot be known quantitatively. For example, the $d$ electrons and $d-d$ transitions of $d$-block metals generate variable redox, magnetic and polyfunctional properties; some elements of this block such as zirconium, manganese, vanadium and tin easily form oxide nanostructures with a very extensive surface area and unique active centers to be used for adsorption and support in heterogeneous catalysis with transition metal compounds. The exact contribution of such centers to $\eta$ cannot be calculated exactly, but one can consider the nature of $\eta$ as the same of the coercive force in materials reversing by DWs motion. The DW energy $E_{w}$ also changes locally due to imperfections. If one considers the largest potential barrier faced by a DW moving along the material and the field $H_{\max }$ necessary to displace it over the barrier, since the magnetization variation is $2 M, H_{\max }$ can be expressed as

$$
H_{\max }=\frac{1}{2 M}\left(\frac{\mathrm{d} E_{w}}{d \xi}\right)_{\max } .
$$

When the DW reaches one of the potential minima, then it moves periodically over a small distance $\xi$, with a restoring force $-\eta \xi$ acting on it due to the imperfections. At the equilibrium position the stiffness is

$$
\eta=\frac{\mathrm{d}^{2} E_{w}}{\mathrm{~d} \xi^{2}} .
$$

Now the three contributions to DW motion can be combined into an equation of motion:

$$
m_{w} \frac{\mathrm{d}^{2} \xi}{\mathrm{d} t^{2}}+\delta \frac{\mathrm{d} \xi}{\mathrm{d} t}+\eta \xi=2 M H
$$

In many empirical cases, the effect of the imperfections is not reversible, so that the inertial term is negligible and the DW motion is over a large distance. In this case Equation (9) becomes

$$
\delta \frac{\mathrm{d} \xi}{\mathrm{d} t}=2 M\left(H-H_{\max }\right)
$$

where $H_{\max }$ is a field required to prevail over the viscous resistance due to defects.

In summary, a new general equation that describes the motion of domain walls in a magnetic thin film in the presence of an external magnetic field has been reported. This must be regarded as the main result contained in this paper. However, a new experimental potential mechanism for DW 
motion, providing a new degree of freedom to control the motion and enabling the development of new optoelectronic memory and logic devices, has been proposed in the introductory paragraph.

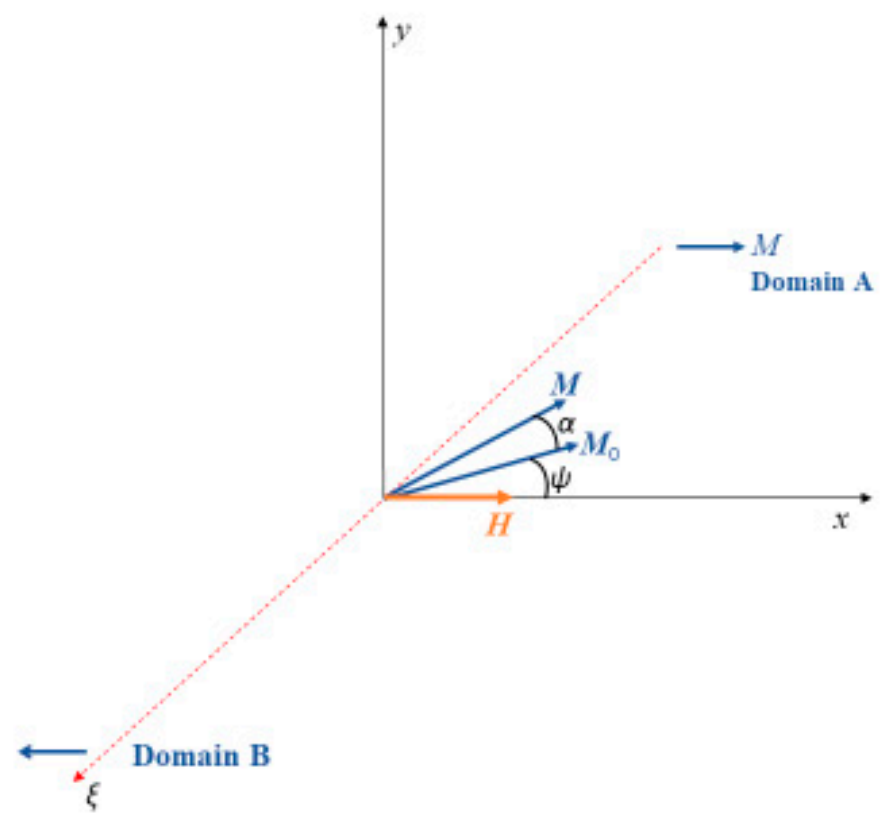

Figure 1. $M_{0}$ is the magnetization at $H=0 ; M$ is the magnetization in applied field.

Funding: This research was funded by FFABR 2017-UNIFE.

Conflicts of Interest: The author declares no conflicts of interest.

\section{References}

1. Raquet, B.; Mamy, R.; Ousset, J.C. Magnetization reversal dynamics in ultrathin magnetic layers. J. Magn. Magn. Mater. 1997, 165, 492-495. [CrossRef]

2. Grollier, J.; Chanthbouala, A.; Matsumoto, R.; Anane, A.; Cros, V.; Nguyen van Dau, F.; Albert Fert, C.R. Magnetic domain wall motion by spin transfer. Physique 2011, 12, 309-317. [CrossRef]

3. Khvalkovskiy, A.V.; Cros, V.; Apalkov, D.; Nikitin, V.; Krounbi, M.; Zvezdin, K.A.; Anane, A.; Grollier, J.; Fert, A. Matching domain-wall configuration and spin-orbit torques for efficient domain-wall motion. Phys. Rev. B 2013, 87, 020402(R). [CrossRef]

4. Depassier, M.C. Speed of domain walls in thin nanotubes: The transition from the linear to the magnonic regime. Phys. Rev. B 2019, 100, 144402. [CrossRef]

5. Van de Wiele, B.; Fin, S.; Sarella, A.; Vavassori, P.; Bisero, D. How finite sample dimensions affect the reversal process of magnetic dot arrays. Appl. Phys. Lett. 2014, 105, 162407. [CrossRef]

6. Bisero, D.; Cremon, P.; Madami, M.; Tacchi, S.; Gubbiotti, G.; Carlotti, G.; Adeyeye, A.O. Magnetization reversal of rectangular particles: Closure states and effect of dipolar coupling. IEEE Trans. Magn. 2012, 48, 1593-1596. [CrossRef]

7. Ranchal, R.; Fin, S.; Bisero, D.; Aroca, C. Tailoring the magnetic anisotropy and domain patterns of sputtered TbFeGa alloys. J. Alloys Compd. 2014, 582, 839-843. [CrossRef]

8. Madami, M.; Bisero, D.; Gubbiotti, G.; Tacchi, S.; Carlotti, G.; Nakano, K.; Ono, T. Magnetization configurations in NiFe slotted rings studied by magneto-optical Kerr effect and Magnetic Force Microscopy. IEEE Trans. Magn. 2012, 48, 1269-1272. [CrossRef]

9. Fernandez-Roldan, J.A.; de Riz, A.; Trapp, B.; Thirion, C.; Vazquez, M.; Toussaint, J.-C.; Fruchart, O.; Gusakova, D. Modeling magnetic-field-induced domain wall propagation in modulated-diameter cylindrical nanowires. Sci. Rep. 2019, 9, 5130. [CrossRef] [PubMed]

10. Sepioni, M.; Madami, M.; Tacchi, S.; Gubbiotti, G.; Carlotti, G.; Bisero, D.; Adeyeye, A.O.; Singh, N.; Goolaup, S. Dipolar interaction in dense chains of submicrometric rectangular dots. J. Phys. Conf. Ser. 2010, 200, 072089. [CrossRef] 
11. Bisero, D.; Cremon, P.; Madami, M.; Sepioni, M.; Tacchi, S.; Gubbiotti, G.; Carlotti, G.; Adeyeye, A.O. Effect of dipolar interaction on the magnetization state of chains of rectangular particles located either head-to-tail or side-by-side. J. Nanopart. Res. 2011, 13, 5691-5698. [CrossRef]

12. Ranchal, R.; Fin, S.; Bisero, D. Magnetic microstructures in electrodeposited $\mathrm{Fe}_{1-\mathrm{x}} \mathrm{Ga}_{\mathrm{x}}$ thin films $(15<\mathrm{x}<22$ at.\%). J. Phys. D 2015, 48, 075001. [CrossRef]

13. Landau, L.; Lifshitz, E. On the theory of the dispersion of magnetic permeability in ferromagnetic bodies. Phys. Z. Sowjet 1935, 8, 153.

14. Cullity, B.D.; Graham, C.D. Introduction to Magnetic Materials, 2nd ed.; Wiley: Hoboken, NJ, USA, 2009.

15. Seitz, F.; Turnbull, D. Solid State Physics; Academic Press: New York, NY, USA, 1956; Volume 3.

(C) 2020 by the author. Licensee MDPI, Basel, Switzerland. This article is an open access article distributed under the terms and conditions of the Creative Commons Attribution (CC BY) license (http://creativecommons.org/licenses/by/4.0/). 